\title{
A nonstandard Volterra integral equation on time scales
}

https://doi.org/10.1515/dema-2019-0042

Received March 30, 2019; accepted November 9, 2019

Abstract: This paper introduces the more general result on existence, uniqueness and boundedness for solutions of nonstandard Volterra type integral equation on an arbitrary time scales. We use Lipschitz type function and the Banach's fixed point theorem at functional space endowed with a suitable Bielecki type norm. Furthermore, it allows to get new sufficient conditions for boundedness and continuous dependence of solutions.

Keywords: Volterra integral equations, time scales, bounded solution, continuous dependence

MSC 2010: 45D05, 45G10, 34N05

\section{Introduction}

This paper introduces the more general result on existence, uniqueness and boundedness for solutions of nonstandard Volterra type integral equation on an arbitrary time scales.

The field of dynamic equations on time scales is an emerging area that has more potential created by Hilger in 1990 [1]. This new and compelling area of mathematics is more general and versatile than the traditional theories of differential and difference equations. The field of dynamic equations on time scales contains and extends the classical theory of differential, difference, integral and summation equations as special cases.

This paper considers nonstandard Volterra type integral equation on an arbitrary time scales $\mathbb{T}$

$$
x(t)=f(t)+\int_{a}^{t} K(t, s, x(s), x(\sigma(s))) \Delta s, \quad a, t \in I_{\mathbb{T}}=[a,+\infty) \cap \mathbb{T},
$$

where $x: I_{\mathbb{T}} \rightarrow \mathbb{R}^{n}$ is the unknown function, $f: I_{\mathbb{T}} \rightarrow \mathbb{R}^{n}$ and $K: I_{\mathbb{T}} \times I_{\mathbb{T}} \times \mathbb{R}^{n} \times \mathbb{R}^{n} \rightarrow \mathbb{R}^{n}$ are nonlinear functions. We will generalize and the same time simplify the results [2-6], find the new sufficient conditions for the existence and uniqueness of solution for nonstandard Volterra type integral equation. We also find condition for boundedness of solutions and prove the continuous dependence of solutions.

T. Kulik and C.C. Tisdell [2] believe that it might be interesting to study the qualitative and quantitative properties of Volterra type integral equations on time scales

$$
x(t)=f(t)+\int_{a}^{t} K(t, s, x(\sigma(s))) \Delta s, \quad a, t \in I_{\mathbb{T}}=[a,+\infty) \cap \mathbb{T} .
$$

\footnotetext{
*Corresponding Author: Andrejs Reinfelds: Institute of Mathematics and Computer Science, University of Latvia, 29 Raina bulvāris, Rīga, LV-1459; Department of Mathematics, University of Latvia, 3 Jelgavas iela, LV-1004, Rīga, Latvia; E-mail: reinf@latnet.lv

Shraddha Christian: Department of Mathematics, University of Latvia, 3 Jelgavas iela, LV-1004, Rīga, Latvia; E-mail: sc16024@lu.lv
} 
This type of integral equations could be very useful for modelling economic process, for example, a KeynesianCross model with "lagged" income [7, 8].

D.B. Pachpatte [3] consider a nonstandard Volterra type dynamic integral equation

$$
x(t)=f(t)+\int_{a}^{t} K\left(t, s, x(s), x^{\Delta}(s)\right) \Delta s, \quad a, t \in I_{\mathbb{T}}=[a,+\infty) \cap \mathbb{T} .
$$

Using the relation

$$
x(\sigma(s))=x(s)+x^{\Delta}(s) \mu(s)
$$

in many cases (2) can be reduced to nonstandard Volterra type integral equation (1).

The main techniques employed are contemporary components of nonlinear analysis, including the Banach's fixed point theorem at appropriate functional space, Lipschitz condition. To understand the notation in this article some basic definitions are needed (for details see $[9,10]$ ).

A time scale $\mathbb{T}$ is an arbitrary non empty closed subset of the real numbers $\mathbb{R}$. Since a time scale may or may not be connected, the concept of jump operator is useful for describing the structure of the time scale under consideration and is also used in defining the delta derivative. The forward jump operator $\sigma: \mathbb{T} \rightarrow \mathbb{T}$ is defined by the equality

$$
\sigma(t)=\inf \{s \in \mathbb{T} \mid s>t\}
$$

while the backward jump operator $\rho: \mathbb{T} \rightarrow \mathbb{T}$ is defined by the equality

$$
\rho(t)=\sup \{s \in \mathbb{T} \mid s<t\} .
$$

The graininess function $\mu: \mathbb{T} \rightarrow[0,+\infty)$ is defined by

$$
\mu(t)=\sigma(t)-t .
$$

The jump operators allow the classification of points in a time scale $\mathbb{T}$. If $\sigma(t)>t$, then the point $t \in \mathbb{T}$ is called right scattered while if $\rho(t)<t$, then the point $t \in \mathbb{T}$ is called left scattered. If $\sigma(t)=t$ then $t \in \mathbb{T}$ is called right dense while if $\rho(t)=t$ then $t \in \mathbb{T}$ is called left dense.

Assume $g: \mathbb{T} \rightarrow \mathbb{R}$ is a function and fix $t \in \mathbb{T}^{K}$. The delta derivative (also Hilger derivative) $g^{\Delta}(t)$ exists if for every $\varepsilon>0$ there exists a neighbourhood $U=(t-\delta, t+\delta) \cap \mathbb{T}$ for some $\delta>0$ such that

$$
\left|(g(\sigma(t))-g(s))-g^{\Delta}(t)(\sigma(t)-s)\right| \leq \varepsilon|\sigma(t)-s|, \text { for all } s \in U .
$$

Take $\mathbb{T}=\mathbb{R}$ and $g$ is differentiable in the ordinary sense at $t \in \mathbb{T}$. Then $g^{\Delta}(t)=g^{\prime}(t)$ is the derivative used in standard calculus. Take $\mathbb{T}=\mathbb{Z}$. Then $g^{\Delta}(t)=\Delta g(t)$ is the forward difference operator used in difference equation.

A function $g: \mathbb{T} \rightarrow \mathbb{R}$ is called rd-continuous provided it is continuous at every right dense points in $\mathbb{T}$ and its left sided limits exist at every left dense points in $\mathbb{T}$.

If $F^{\Delta}(t)=g(t)$ then define the (Cauchy) delta integral by

$$
\int_{r}^{s} g(t) \Delta t=F(s)-F(r), \text { for all } r, s \in \mathbb{T} .
$$

If $\mathbb{T}=\mathbb{R}$, then

$$
\int_{r}^{s} g(t) \Delta t=\int_{r}^{s} g(t) d t
$$

while $\mathbb{T}=\mathbb{Z}$, then

$$
\int_{r}^{s} g(t) \Delta t=\sum_{t=r}^{s-1} g(t), \text { if } r, s \in \mathbb{Z} \text { and } r<s .
$$




\section{Existence and uniqueness of solution on time scales}

Consider the integral equation (1). We now construct the appropriate Banach space for our analysis. Let $\beta: I_{\mathbb{T}} \rightarrow \mathbb{R}$ be a nonnegative and rd-continuous scalar function, where $I_{\mathbb{T}}=[a,+\infty) \cap \mathbb{T}$. The Cauchy initial value problem for scalar linear equation

$$
x^{\Delta}=\beta(t) x, \quad x(a)=1
$$

has the unique solution $e_{\beta}(\cdot, a): I_{\mathbb{T}} \rightarrow \mathbb{R}$ [9]. More explicitly, using the cylinder transformation the generalized exponential function $e_{\beta}(\cdot, a)$ is given by

$$
e_{\beta}(t, a)= \begin{cases}\exp \left(\int_{a}^{t} \beta(s) d s\right), & \text { for } \mu(s)=0, \\ \exp \left(\int_{a}^{t} \frac{\ln (1+\mu(s) \beta(s))}{\mu(s)} \Delta s\right), & \text { for } \mu(s)>0,\end{cases}
$$

where $\ln$ is the principal logarithm function. We will use the following property of exponential function [9]

$$
e_{\beta}(\sigma(t), a)=(1+\mu(t) \beta(t)) e_{\beta}(t, a) .
$$

Observe that we also have Bernoulli’s type inequality [11]

$$
1 \leq 1+\int_{a}^{t} \beta(s) \Delta s \leq e_{\beta}(t, a) \leq \exp \left(\int_{a}^{t} \beta(s) \Delta s\right)
$$

for all $t \in I_{\mathbb{T}}$.

Let $|\cdot|$ denote the Euclidean norm on $\mathbb{R}^{n}$. We will consider the linear space of continuous functions $C\left(I_{\mathbb{T}} ; \mathbb{R}^{n}\right)$ such that

$$
\sup _{t \in I_{\mathbb{T}}} \frac{|x(t)|}{e_{\beta}(t, a)}<\infty
$$

and denote this special space by $C_{\beta}\left(I_{\mathbb{T}} ; \mathbb{R}^{n}\right)$. The space $C_{\beta}\left(I_{\mathbb{T}} ; \mathbb{R}^{n}\right)$ can be endowed with a suitable Bielecki type norm, expressly

$$
\|x\|_{\beta}=\sup _{t \in I_{\mathbb{T}}} \frac{|x(t)|}{e_{\beta}(t, a)} .
$$

We generalize and at the same time simplify the results [2-6] assuming $L_{1}$ and $L_{2}$ can be an unbounded rd-continuous functions. In the case of unbounded time scale, the Lipschitz type functions $L_{1}$ and $L_{2}$ could be unbounded. The use of Bielecki type norms related to Lipschitz type functions allows to choose a suitable functional space to prove the following theorem.

Theorem 1. Consider the integral equation (1). Let $K: I_{\mathbb{T}} \times I_{\mathbb{T}} \times \mathbb{R}^{n} \times \mathbb{R}^{n} \rightarrow \mathbb{R}^{n}$ be continuous in its first, third and fourth variables and rd-continuous in its second variable, $f: I_{\mathbb{T}} \rightarrow \mathbb{R}^{n}$ be continuous, $L_{1}, L_{2}: I_{\mathbb{T}} \rightarrow \mathbb{R}$ be $r d$-continuous, $\sup _{s \in I_{\mathbb{T}}}\left|L_{2}(s) \mu(s)\right|=r<1,1<\gamma<r^{-1}$ and $\beta(s)=\frac{\left[L_{1}(s)+L_{2}(s)\right] \gamma}{1-r \gamma}$. If

$$
\begin{gathered}
|K(t, s, p, \bar{p})-K(t, s, q, \bar{q})| \leq L_{1}(s)|p-q|+L_{2}(s)|\bar{p}-\bar{q}|, p, q, \bar{p}, \bar{q} \in \mathbb{R}^{n}, s<t, \\
m=\sup _{t \in I_{T}} \frac{1}{e_{\beta}(t, a)}\left|f(t)+\int_{a}^{t} K(t, s, 0,0) \Delta s\right|<\infty,
\end{gathered}
$$

then the integral equation (1) has a unique solution $x \in C_{\beta}\left(I_{\mathbb{T}} ; \mathbb{R}^{n}\right)$.

Proof. Consider the following equivalent formulation of (1), namely

$$
x(t)=\left(f(t)+\int_{a}^{t} K(t, s, 0,0) \Delta s\right)+\int_{a}^{t}(K(t, s, x(s), x(\sigma(s)))-K(t, s, 0,0)) \Delta s .
$$


We will show that (7) has a unique solution and thus (1) must also have a unique solution.

Let $L_{1}, L_{2}: I_{\mathbb{T}} \rightarrow \mathbb{R}$ be the functions defined in (5) and let $\beta(s)=\frac{\left[L_{1}(s)+L_{2}(s)\right] \gamma}{1-\gamma r}$, where $1<\gamma<r^{-1}$. From (3) follows

$$
\begin{aligned}
\operatorname{ff}_{1}(s)+L_{2}(s)(1+\mu(s) \beta(s)) & =L_{1}(s)+L_{2}(s)+L_{2}(s) \mu(s) \beta(s) \\
& \leq L_{1}(s)+L_{2}(s)+\frac{r\left[L_{1}(s)+L_{2}(s)\right] \gamma}{1-r \gamma} \\
& =\frac{L_{1}(s)+L_{2}(s)}{1-r \gamma}=\frac{\beta(s)}{\gamma} .
\end{aligned}
$$

Consider the Banach space $C_{\beta}\left(I_{\mathbb{T}} ; \mathbb{R}^{n}\right)$ and let operator $F$ be defined by

$$
[F x](t)=\left(f(t)+\int_{a}^{t} K(t, s, 0,0) \Delta s\right)+\int_{a}^{t}(K(t, s, \chi(s), \chi(\sigma(s)))-K(t, s, 0,0)) \Delta s .
$$

Fixed point of $F$ will be solution to (7). Thus we want to prove that there exists a unique $x$ such that $F x=x$. We show that $F: C_{\beta}\left(I_{\mathbb{T}} ; \mathbb{R}^{n}\right) \rightarrow C_{\beta}\left(I_{\mathbb{T}} ; \mathbb{R}^{n}\right)$. Let $x \in C_{\beta}\left(I_{\mathbb{T}} ; \mathbb{R}^{n}\right)$. Taking norms in (7), we obtain

$$
\begin{aligned}
& \|F x\|_{\beta}=\sup _{t \in I_{\mathbb{T}}} \frac{1}{e_{\beta}(t, a)} \mid f(t)+\int_{a}^{t} K(t, s, 0,0) \Delta s \\
& +\int_{a}^{t}(K(t, s, x(s), x(\sigma(s)))-K(t, s, 0,0)) \Delta s \\
& \leq m+\sup _{t \in I_{\mathbb{T}}} \frac{1}{e_{\beta}(t, a)} \int_{a}^{t}\left[L_{1}(s)|x(s)|+L_{2}(s)|x(\sigma(s))|\right] \Delta s \\
& =m+\sup _{t \in I_{\mathbb{T}}} \frac{1}{e_{\beta}(t, a)} \int_{a}^{t}\left[L_{1}(s) e_{\beta}(s, a) \frac{|x(s)|}{e_{\beta}(s, a)}+L_{2}(s) e_{\beta}(\sigma(s), a) \frac{|x(\sigma(s))|}{e_{\beta}(\sigma(s), a)}\right] \Delta s \\
& \leq m+\|x\|_{\beta} \sup _{t \in I_{\mathbb{T}}} \frac{1}{e_{\beta}(t, a)} \int_{a}^{t}\left[L_{1}(s)+L_{2}(s)(1+\mu(s) \beta(s))\right] e_{\beta}(s, a) \Delta s \\
& =m+\frac{\|x\|_{\beta}}{\gamma} \sup _{t \in I_{\mathbb{T}}} \frac{1}{e_{\beta}(t, a)} \int_{a}^{t} \beta(s) e_{\beta}(s, a) \Delta s \\
& =m+\frac{\|x\|_{\beta}}{\gamma} \sup _{t \in I_{\mathbb{T}}} \frac{1}{e_{\beta}(t, a)} \int_{a}^{t} e_{\beta}^{\Delta}(s, a) \Delta s \\
& =m+\frac{\|x\|_{\beta}}{\gamma} \sup _{t \in I_{\mathbb{T}}} \frac{1}{e_{\beta}(t, a)}\left(e_{\beta}(t, a)-1\right) \\
& =m+\frac{\|x\|_{\beta}}{\gamma} \sup _{t \in I_{\mathbb{T}}}\left(1-\frac{1}{e_{\beta}(t, a)}\right) \leq m+\frac{\|x\|_{\beta}}{\gamma}<\infty \text {. }
\end{aligned}
$$

Hence we see that $F: C_{\beta}\left(I_{\mathbb{T}} ; \mathbb{R}^{n}\right) \rightarrow C_{\beta}\left(I_{\mathbb{T}} ; \mathbb{R}^{n}\right)$. We show that $F$ is a contraction map with contraction constant $\alpha=1 / \gamma<1$ and then Banach's fixed point theorem will apply.

For any $u, v \in C_{\beta}\left(I_{\mathbb{T}} ; \mathbb{R}^{n}\right)$

$$
\begin{aligned}
\|F u-F v\|_{\beta} & =\sup _{t \in I_{\mathbb{T}}} \frac{|[F u](t)-[F v](t)|}{e_{\beta}(t, a)} \\
& \leq \sup _{t \in I_{\mathbb{T}}} \frac{1}{e_{\beta}(t, a)} \int_{a}^{t}|K(t, s, u(s), u(\sigma(s)))-K(t, s, v(s), v(\sigma(s)))| \Delta s
\end{aligned}
$$




$$
\begin{aligned}
& \leq \sup _{t \in I_{\mathbb{T}}} \frac{1}{e_{\beta}(t, a)} \int_{a}^{t}\left[L_{1}(s)|u(s)-v(s)|+L_{2}(s)|u(\sigma(s))-v(\sigma(s))|\right] \Delta s \\
& =\sup _{t \in I_{\mathbb{T}}} \frac{1}{e_{\beta}(t, a)} \int_{a}^{t}\left[L_{1}(s) e_{\beta}(s, a) \frac{|u(s)-v(s)|}{e_{\beta}(s, a)}+L_{2}(s) e_{\beta}(\sigma(s), a) \frac{|u(\sigma(s))-v(\sigma(s))|]}{e_{\beta}(\sigma(s), a)}\right] \Delta s \\
& \leq \quad\|u-v\|_{\beta} \sup _{t \in I_{\mathbb{T}}} \frac{1}{e_{\beta}(t, a)} \int_{a}^{t}\left[L_{1}(s)+L_{2}(s)(1+\mu(s) \beta(s))\right] e_{\beta}(s, a) \Delta s \\
& =\frac{\|u-v\|_{\beta}}{\gamma} \sup _{t \in I_{\mathbb{T}}} \frac{1}{e_{\beta}(t, a)} \int_{a}^{t} \beta(s) e_{\beta}(s, a) \Delta s \\
& =\frac{\|u-v\|_{\beta}}{\gamma} \sup _{t \in I_{\mathbb{T}}} \frac{1}{e_{\beta}(t, a)} \int_{a}^{t} e_{\beta}^{\Delta}(s, a) \Delta s \\
& =\frac{\|u-v\|_{\beta}}{\gamma} \sup _{t \in I_{\mathbb{T}}} \frac{1}{e_{\beta}(t, a)}\left(e_{\beta}(t, a)-1\right) \\
& =\frac{\|u-v\|_{\beta}}{\gamma} \sup _{t \in I_{\mathbb{T}}}\left(1-\frac{1}{e_{\beta}(t, a)}\right) \leq \frac{\|u-v\|_{\beta}}{\gamma}=\alpha\|u-v\|_{\beta} .
\end{aligned}
$$

As $\alpha<1$, we see that $F$ is a contraction map and so Banach's fixed point theorem applies, yielding the existence of a unique fixed point $x$ of $F$.

Example. Consider the scalar integral equation

$$
x(t)=t^{2}+\int_{a}^{t}(s+\sigma(s))\left[x(\sigma(s))^{2}+1\right]^{\frac{1}{2}} \Delta s, \quad a, t \in I_{\mathbb{T}}=[a,+\infty) \cap \mathbb{T}, a \geq 0 .
$$

We claim that this integral equation has a unique solution if

$$
\sup _{s \in I_{\mathbb{T}}}|(s+\sigma(s)) \mu(s)|=r<1
$$

for an arbitrary time scales $\mathbb{T}$.

Proof. We will use the above Theorem 1 and make use of the fact that $K(t, s, p)=(s+\sigma(s))\left(p^{2}+1\right)^{\frac{1}{2}}$ has a bounded partial derivative with respect to $p$ everywhere consider

$$
\begin{aligned}
|K(t, s, p)-K(t, s, q)| & =\left|(s+\sigma(s))\left(p^{2}+1\right)^{\frac{1}{2}}-(s+\sigma(s))\left(q^{2}+1\right)^{\frac{1}{2}}\right| \\
& \leq(s+\sigma(s)) \sup _{r \in \mathbb{R}}\left|\frac{r}{\left(r^{2}+1\right)^{\frac{1}{2}}}\right||p-q| \\
& \leq(s+\sigma(s))|p-q| .
\end{aligned}
$$

Here we used the mean value theorem. So (5) holds with $L_{2}(s)=s+\sigma(s)=2 s+\mu(s)$. It is not difficult to verify that (6) holds. Using the Bernoulli's inequality (4) we get

$$
e_{\beta}(t, a) \geq 1+\frac{\gamma\left(t^{2}-a^{2}\right)}{1-r \gamma}
$$

is followed by the estimate $m<\infty$. The result now follows from Theorem 1 .

\section{Bounded solution}

We now present results concerning boundedness of solutions of nonstandard Volterra type integral equations. 
Theorem 2. Consider the nonstandard Volterra type integral equation (1) satisfying conditions of Theorem 1. If in addition

$$
\sup _{t \in I_{\mathbb{T}}} \int_{a}^{t}\left(L_{1}(s)+L_{2}(s)\right) \Delta s=v<\infty
$$

and

$$
m_{1}=\sup _{t \in I_{\mathbb{T}}}\left|f(t)+\int_{a}^{t} K(t, s, 0,0) \Delta s\right|<\infty,
$$

then the unique solution of nonstandard Volterra type integral equation (1) on unbounded above time scales is bounded

$$
\sup _{t \in I_{\mathbb{T}}}|x(t)|<+\infty
$$

Proof. In our case $\beta(s)=\frac{\left[L_{1}(s)+L_{2}(s)\right] \gamma}{1-r \gamma}$. Using the estimate (4) and condition (8) we get that

$$
1 \leq e_{\beta}(t, a) \leq \exp \left(\int_{a}^{t} \beta(s) \Delta s\right) \leq \exp \left(\frac{\gamma v}{1-r \gamma}\right) .
$$

So norm $\|x\|_{\beta}$ and supremum norm $\sup _{t \in I_{\mathbb{T}}}|\chi(t)|$ are equivalent at space $C_{\beta}\left(I_{\mathbb{T}} ; \mathbb{R}^{n}\right)$. This means that nonstandard Volterra's type integral equation solution is bounded if additional conditions (8) and (9) are fulfilled and $v>0$.

Example. Consider the scalar integral equation

$$
x(t)=2+\int_{a}^{t} \frac{\left(x(\sigma(s))^{2}+1\right)^{\frac{1}{2}}}{s \sigma(s)} \Delta s, \quad a, t \in I_{\mathbb{T}}=[a,+\infty) \cap \mathbb{T}, a>0 .
$$

We claim that this integral equation has bounded solution if

$$
\sup _{s \in I_{\mathbb{T}}}\left|\frac{\mu(s)}{s \sigma(s)}\right|=r<1
$$

for arbitrary unbounded above $\mathbb{T}$.

Proof. Let us note that for $0<a \leq t$

$$
\int_{a}^{t} \frac{\Delta s}{s \sigma(s)}=a^{-1}-t^{-1}<a^{-1}
$$

The result now follows from Theorem 2 .

\section{Continuity of solutions}

We will generalize and simplify the results [4, 6] for nonstandard Volterra integral equation (1) on time scales $\mathbb{T}$. Let $x: I_{\mathbb{T}} \rightarrow \mathbb{R}^{n}$ and $y: I_{\mathbb{T}} \rightarrow \mathbb{R}^{n}$ be the solutions of the nonstandard Volterra integral equations

$$
x(t)=f_{1}(t)+\int_{a}^{t} K(t, s, x(s), x(\sigma(s))) \Delta s,
$$

and

$$
y(t)=f_{2}(t)+\int_{a}^{t} K(t, s, y(s), y(\sigma(s))) \Delta s .
$$


Theorem 3. Consider the nonstandard Volterra integral equations (10) and (11) satisfying conditions of Theorem 1. Let $x(t)$ and $y(t)$ be the solutions of appropriate equations (10), (11) and let $\delta=\left\|f_{1}-f_{2}\right\|_{\beta}$. Then

$$
\|x(t)-y(t)\|_{\beta} \leq \frac{\gamma}{\gamma-1}\left\|f_{1}-f_{2}\right\|_{\beta} .
$$

Proof. Analogous to Theorem 1, we estimate the difference $\left\|f_{1}-f_{2}\right\|_{\beta}$. We get

$$
\|x-y\|_{\beta} \leq\left\|f_{1}-f_{2}\right\|_{\beta}+\frac{\|x-y\|_{\beta}}{\gamma} .
$$

So, we have

$$
\|x-y\|_{\beta} \leq \frac{\gamma}{\gamma-1}\left\|f_{1}-f_{2}\right\|_{\beta}
$$

Example. Let us consider scalar Volterra integral equations

$$
x(t)=f_{1}(t)+\int_{1}^{t} \frac{\left(x(\sigma(s))^{2}+1\right)^{\frac{1}{2}}}{s \sigma(s)} \Delta s \quad \text { and } \quad y(t)=f_{2}(t)+\int_{1}^{t} \frac{\left(x(\sigma(s))^{2}+1\right)^{\frac{1}{2}}}{s \sigma(s)} \Delta s, t \in \mathbb{T},
$$

where the time scale $\mathbb{T}$ is the union of closed intervals

$$
\mathbb{T}=\bigcup_{n \in \mathbb{N}}[n, n+1 / 2]
$$

and $f_{1}, f_{2}: \mathbb{T} \rightarrow \mathbb{R}$ are continuous and bounded functions. Then the graininess function $\mu$ for right dense points is $\mu(t)=0$ and $\mu(t)=1 / 2$ for right scattered points. Note that Lipschitz type function is

$$
L_{2}(s)=\frac{1}{s \sigma(s)}
$$

and observe that

$$
r=\sup _{s \in \mathbb{T}}\left|L_{2}(s) \mu(s)\right|=\frac{2}{9} .
$$

Note that we put $\gamma=2$ and $\beta(s)=3,6 L_{2}(s)$, then

$$
1+\mu(s) \beta(s) \leq 1,8 .
$$

We obtain

$$
\begin{aligned}
|x(t)-y(t)| & \leq\left|f_{1}(t)-f_{2}(t)\right|+\int_{1}^{t} \frac{|x(\sigma(s))-y(\sigma(s))|}{s \sigma(s)} \Delta s \\
& \leq\left|f_{1}(t)-f_{2}(t)\right|+\|x-y\|_{\beta} \int_{1}^{t} \frac{e_{\beta}(\sigma(s), 1)}{s \sigma(s)} \Delta s \\
& =\left|f_{1}(t)-f_{2}(t)\right|+\|x-y\|_{\beta} \int_{1}^{t} \frac{(1+\mu(s) \beta(s)) e_{\beta}(s, 1)}{s \sigma(s)} \Delta s \\
& \leq\left|f_{1}(t)-f_{2}(t)\right|+\frac{\|x-y\|_{\beta}}{2} \int_{1}^{t} \beta(s) e_{\beta}(s, 1) \Delta s \\
& \leq\left|f_{1}(t)-f_{2}(t)\right|+\frac{\|x-y\|_{\beta}}{2}\left(e_{\beta}(t, 1)-1\right) .
\end{aligned}
$$

Hence

$$
\frac{|x(t)-y(t)|}{e_{\beta}(t, 1)} \leq \frac{\left|f_{1}(t)-f_{2}(t)\right|}{e_{\beta}(t, 1)}+\frac{\|x-y\|_{\beta}}{2} .
$$


It follows

$$
\|x(t)-y(t)\|_{\beta} \leq\left\|f_{1}(t)-f_{2}(t)\right\|_{\beta}+\frac{\|x(t)-y(t)\|_{\beta}}{2}
$$

or

$$
\|x-y\|_{\beta} \leq 2\left\|f_{1}-f_{2}\right\|_{\beta} .
$$

Let us note that

$$
1 \leq e_{\beta}(t, 1) \leq \exp \left(\int_{1}^{t} \beta(s) \Delta s\right) \leq e^{3,6} .
$$

It follows that

$$
e^{-3,6} \sup _{t \in \mathbb{T}}|x(t)-y(t)| \leq\|x-y\|_{\beta} \leq \sup _{t \in \mathbb{T}}|x(t)-y(t)| .
$$

So, we get estimate

$$
\sup _{t \in \mathbb{T}}|x(t)-y(t)| \leq 2 e^{3,6} \sup _{t \in \mathbb{T}}\left|f_{1}(t)-f_{2}(t)\right| .
$$

\section{References}

[1] Hilger S., Analysis on measure chains. A unified approach to continuous and discrete calculus, Results Math., 1990, 18, 18-56

[2] Kulik T., Tisdeil C.C., Volterra integral equations on time scales. Basic qualitative and quantitative results with applications to initial value problems on unbounded domains, Int. J. Difference Equ., 2008, 3, 103-133

[3] Pachpatte D.B., On a nonstandard Volterra type dynamic integral equation on time scales, Electron. J. Qual. Theory Differ. Equ., 2009, 72, 1-14

[4] Pachpatte D.B., Properties of some dynamic integral equation on time scales, Ann. Funct. Anal., 2013, 4(2), 12-26

[5] Reinfelds A., Christian S., Volterra integral equations on unbounded time scales, Int. J. Difference Equ., 2019, 14, 169-177

[6] dos Santos I.L.D., On Volterra integral equations on time scales, Mediterr. J. Math., 2015, 12(2), 471-480

[7] Ferguson B.S., Lim G.C., Dynamic Economic Models in Discrete Time. Theory and Empirical Applications, Routledge, London, 2003

[8] Tisdeil C.C., Zaidi A., Basic qualitative and quantitative results for solutions to nonlinear dynamic equations on time scales with an application to economic modelling, Nonlinear Analysis, 2008, 68, 3504-3524

[9] Bohner M., Peterson A., Dynamic Equations on Time Scales. An Introduction with Applications, Birkhäuser, Boston/Basel/Berlin, 2001

[10] Bohner M., Peterson A., Advances in Dynamic Equations on Time Scales, Birkhäuser, Boston/Basel/Berlin, 2003

[11] Bohner M., Some oscillation criteria for first order delay dynamic equations, Far East Journal of Applied Mathematics, 2005, $18,289-304$ 